\title{
A 57 Year-old Man With Prolonged Shortness of Breath AND FEVERS
}

\author{
Loren Chen, $M D$
}

\section{Case Presentation}

A 57 year-old Caucasian male was initially admitted to Methodist Hospital from an outside hospital for dehydration, increased heart rate, and weight loss. He has a past medical history of coronary artery disease status post myocardial infarction and coronary artery bypass graft, congestive heart failure, paroxysmal atrial fibrillation on coumadin, diabetes type II on insulin, and an orthotopic liver transplant in 1997 for end-stage liver disease secondary to hepatitis $\mathrm{C}$ virus and alpha- 1 antitrypsin disorder.

At the outside hospital, his one-month hospital course was complicated by acute on chronic renal failure which warranted hemodialysis, anemia that required transfusions, MRSA line sepsis from his Perm-A-Cath treated with vancomycin, and pneumonia, which was possibly secondary to aspiration. The patient was transferred to our facility for further management.

Additional past medical history is significant for hyperlipidemia, depression and $\mathrm{C} 3$ to $\mathrm{C} 5$ fracture. Additional surgeries include AICD placement, a C3 to C4 diskectomy, cholecystectomy and appendectomy. Social history was negative for alcohol and substance abuse, but positive for a 30 pack year smoking history, which he quit one year ago. Family history was significant for diabetes, coronary artery disease and lung cancer. Current medications included amiodarone, aspirin, carvedilol, nexium, zetia, insulin, nebulizers, sirolimus, vancomycin, zosyn, and gentamicin. His only allergy was to meperidine.

Initial vital signs were temperature $98.9^{\circ}$ Fahrenheit, pulse 77 beats/minutes, respirations 16 breaths/minute, blood pressure $126 / 70 \mathrm{~mm} \mathrm{Hg}$, oxygen saturation $99 \%$ on room air. The patient was in no acute distress. Physical exam was significant only for bilateral crackles at the lung bases, multiple skin tattoos, a rightsided Perm-A-Cath in the chest that was non-tender without surrounding erythema or swelling, and a surgical scar in the right upper quadrant.

Chest radiograph on admission showed a right lower lobe consolidation, and he was continued on antibiotics for presumed pneumonia. The patient appeared to be improving after finishing his course of antibiotics for line sepsis and suspected pneumonia, and plans were made to transfer him to a subacute rehab with outpatient dialysis. One week after hospital transfer, he developed fevers and chills with accompanying shortness of breath. Blood cultures grew out pan-sensitive E. coli from his Perm-A-Cath. The catheter was removed and a Shiley catheter was placed. After completing a course of antibiotics, he remained afebrile, and when his blood cultures showed no growth for 48 hours, another Perm-A-Cath was placed. However, he became febrile again. A repeat chest radiograph showed increasing left lingular pneumonia in addition to an unchanged right lower lobe consolidation (Figure 1). At this point, he finished a course of empiric moxifloxacin which was recommended by the infectious disease service.
He continued to have intermittent fevers, shortness of breath, a non-productive cough, and hypoxia that required oxygen therapy. Prior to this prolonged hospitalization, the patient was able to breath on room air. Serial chest radiographs showed increasing pulmonary infiltrates despite treatment with a triple antibiotic regimen. At this time, physical exam was only remarkable for bibasilar, course crackles, but no wheezes or rhonchi. He had a trans-thoracic echocardiogram that was negative for endocarditis, a CT of the abdomen and pelvis that was negative for abscess, and a chest CT that showed persisting bilateral lower lobe pulmonary opacities, left greater than right, and scattered abnormal mediastinal lymph nodes (Figure 2). Cultures were negative since his episode of E. coli bacteremia, and labs were unremarkable.

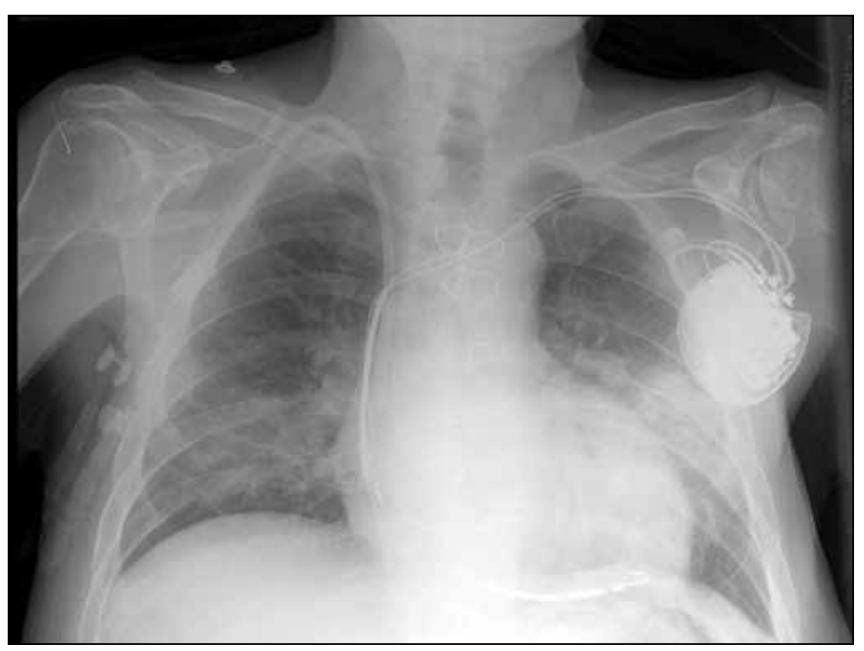

Figure 1. Chest radiograph shows persistent, asymetric, bilateral parenchymal infiltrates without change from two weeks prior. No effusions noted.

A bronchoscopy showed normal airways and no endobronchial lesions. Biopsies and bronchial alveolar lavage (BAL) were taken from the superior segment of the left lingua. The BAL revealed numerous foamy, hemosiderin-laden macrophages, and the biopsy revealed interstitial pneumonitis consistent with amiodarone-induced lung toxicity. Stains were negative for malignant cells.

Amiodarone and antibiotics were stopped, and the patient was started on prednisone $50 \mathrm{mg}$ PO once daily $(1 \mathrm{mg} / \mathrm{kg} /$ day) with a taper to $20 \mathrm{mgs}$ per day as his infiltrates cleared. Within one week of treatment, his fevers resolved with near resolution of his respiratory symptoms by the time of transfer to rehab. His vasculitis labs including ANCA, ANA, ESR, double-stranded DNA, anti-Jo, RF, anti-GBM and IgA, were negative. Further tests for Legionella, AFB, respiratory viruses, hepatitis B, HIV, CMV, and EBV were negative. In regards to his atrial fibrillation, since amiodarone was discontinued, his carvedilol was increased, and 

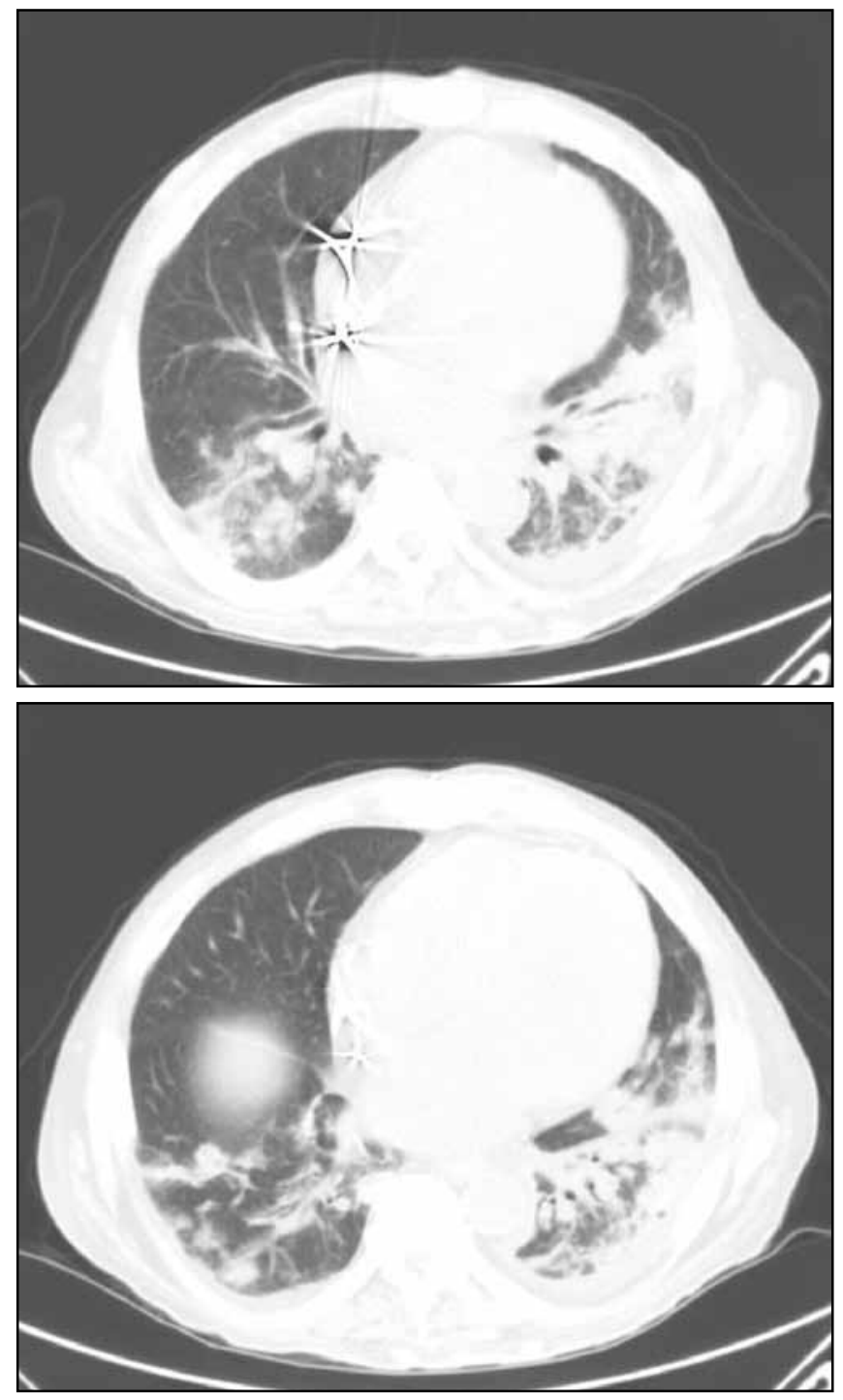

Figure 2. Chest CT shows persistent bilateral lower lobe pulmonary opacities, left greater than right, extending to the periphery.

the patient remained in sinus rhythm throughout the rest of his hospitalization.

A follow-up chest CT two months later revealed significant improvement of his infiltrates and ground glass opacities.

\section{Discussion}

Although amiodarone is approved by the Food and Drug Administration only for refractory ventricular arrhythmias, it is one of the most frequently prescribed antiarrhythmic medications in the United States. ${ }^{1}$ According to a large evidencebased review, amiodarone has clinical value in patients with left ventricular dysfunction and heart failure as first-line treatment
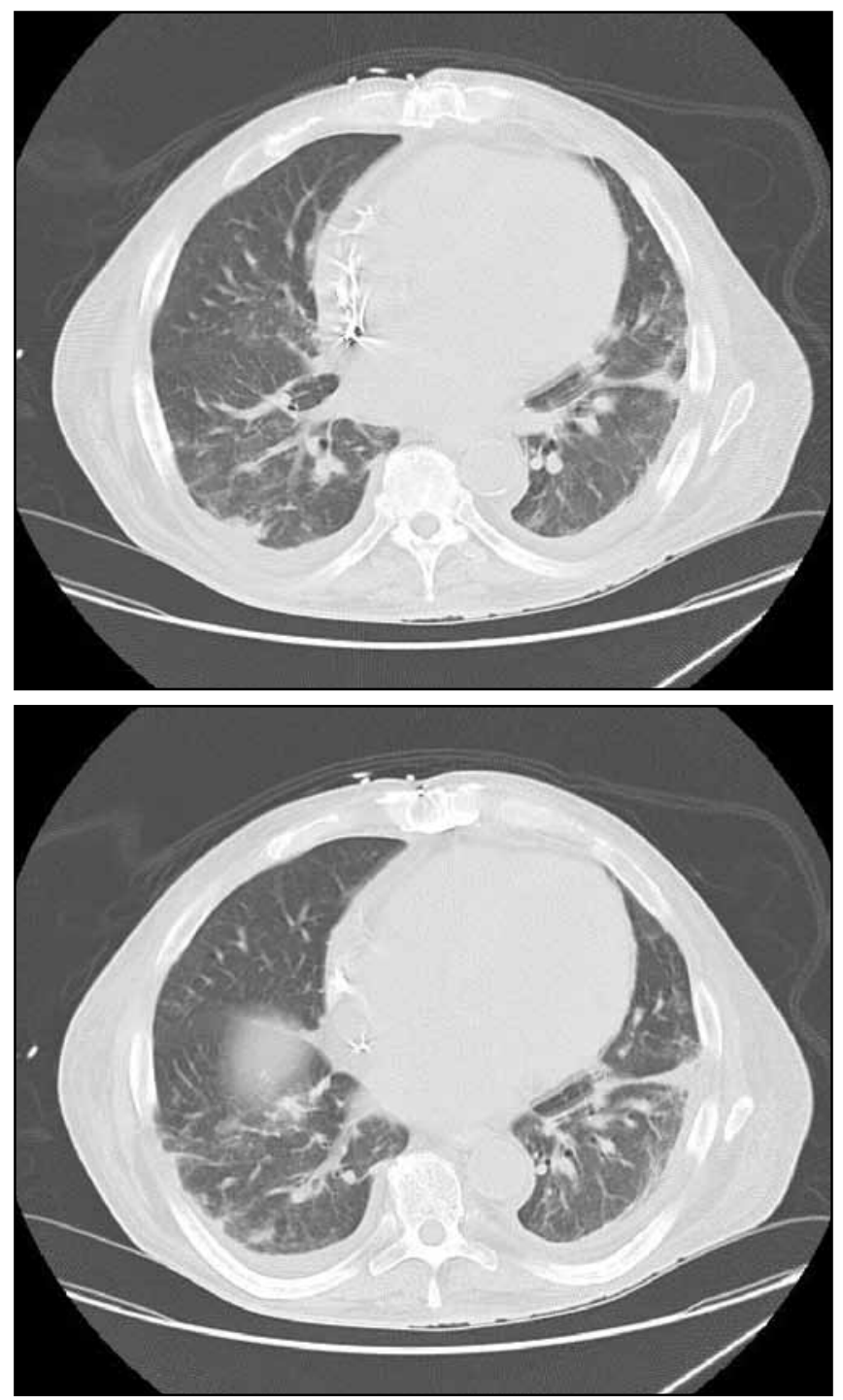

Figure 3. Two month follow-up chest CT shows resolving infiltrates and ground glass opacities.

for atrial fibrillation and acute management of sustained ventricular tachyarrythmias. ${ }^{2}$ It can have a prophylactic role during the perioperative period of cardiac surgery and may be an effective adjunct to implantable cardioverter-defibrillator therapy to reduce the number of shocks. However, adverse side effects are common and include corneal microdeposits (>90\%), optic neuropathy/neuritis $(<1 \%$ to $2 \%)$, blue-gray skin discoloration ( $4 \%$ to $9 \%)$, photosensitivity $(25 \%$ to $75 \%)$, hypothyroidism (6\%), hyperthyroidism ( $0.9 \%$ to $2 \%)$, peripheral neuropathy $(0.3 \%)$, hepatotoxicity (15\% to $30 \%)$, hepatitis and cirrhosis $(<3 \%)$, and pulmonary toxicity $(1 \%$ to $7 \%){ }^{2}$ 
Amiodarone was initially developed in the 1960s as an antianginal agent due to its vasodilatory properties and serendipitously was found to suppress arrythmias. Despite widespread use for nearly two decades in Western Europe, there were no reported cases of associated pulmonary toxicity until 1980, after it was introduced as an investigational agent in this country. This was initially thought to be due to the higher doses used for ventricular arrythmias, ${ }^{3}$ but retrospective analysis revealed that cases had been observed in Europe, but were missed. Currently, amiodarone-induced pulmonary toxicity (APT) carries a mortality between $21 \%$ to $33 \%$ of patients who are admitted to the hospital. ${ }^{4}$

The cause of APT is not well understood. Amiodarone and its metabolite, desethyl-amiodarone (DEAm), are amphiphilic cations which accumulate in tissues, including the lung. It can also infiltrate into the liver, skin, thyroid, and eye, which are other common sites for adverse effects. In regards to pulmonary toxicity, amiodarone can damage lung tissue directly via a cytotoxic process and indirectly via immunological reactions, supported by findings of CD-8-positive, cytotoxic T cells on bronchoalveolar lavage often in combination with polymorphonuclear cells, increased production of toxic oxygen radicals, and accumulation of phospholipids complexes which interfere with normal cellular metabolic pathways., 5, 6

Risk factors for developing APT are increased age, pre-existing lung disease, recent pulmonary insults, cardiopulmonary surgery involving oxygen given at high concentrations, and dosages greater than $400 \mathrm{mg} /$ day. It is more frequent in men and unusual in patients younger than 40 years old, though rare case have been described in children. Most amiodarone-induced pulmonary manifestations are found to occur when the dosage exceeds $400 \mathrm{mg} /$ day administered for more than 2 months or when a lower dosage is given for more than 2 years. ${ }^{2,4,5}$ However, APT has been noted to occur in lower doses, albeit not as frequently. ${ }^{7}$ On average, pulmonary toxicity will develop in $5 \%$ to $15 \%$ of patients who take $500 \mathrm{mg} /$ day or more and $0.1 \%$ to $0.5 \%$ who take up to $200 \mathrm{mg} /$ day. ${ }^{4}$

The diagnosis of APT is one of exclusion including, but not limited to heart failure, infectious pneumonia, organizing pneumonia, pulmonary embolism, pulmonary infarct, and malignancy. Onset of pneumonitis can develop at any time from a few days after an initial loading dose to more than a decade into treatment. Signs and symptoms are generally nonspecific and include nonproductive cough, dyspnea, fever, weight loss, and pleuritic chest pain.

Physical exam typically reveals bilateral rales. Patients with APT may have nonspecific elevations in WBC, LDH, and ESR. Amiodarone levels or DEAm have not been shown to be predictive or diagnostic of APT. Pulmonary Function Testing usually demonstrates typical changes of restrictive lung disease and reduced diffusing capacity of the lung for carbon monoxide
(D $\mathrm{CO})$, but most patients on amiodarone already have abnormal PFTs. ${ }^{2}$

Chest radiograph may reveal diffuse or localized interstitial, alveolar, or mixed opacities, and often presents with an asymmetrical pattern. It can also be accompanied by pleural thickening, especially if the opacities are localized in the periphery, pleural effusion, or a mass (or masses). Acute Respiratory Distress Syndrome (ARDS) can occur in patients treated with amiodarone almost exclusively following thoracic surgery. Diuresis that does not result in clearing of pulmonary infiltrates should increase consideration of APT. ${ }^{2,4}$ Most progressing lung parenchymal disorders will eventually be evaluated by a bronchoscopic evaluation.

In the setting of APT, on bronchoalveolar lavage, the presence of foam cells with lamellar inclusions in patients chronically exposed to amiodarone is a routine finding and dose not indicate drug toxicity. However, the absence of foam cells makes APT highly unlikely. A lung biopsy specimen of substantial size maybe required to establish a more certain diagnosis and would reveal parenchymal lung changes consistent with nonspecific interstitial pneumonitis, organizing pneumonia, or diffuse alveolar damage. It may also be useful in revealing another potential cause of pulmonary injury, such as malignancy. However, since ARDS can develop in patients on amiodarone following thoracic surgery, open or thoracoscopic lung biopsies are indicated when an accurate diagnosis is required early for example, in patients in whom amiodarone cannot be held without risks, if a diagnosis of pulmonary opacities is required prior to heart surgery, and in patients who do not improve by one to two months after drug cessation and initiation of steroids to evaluate for other causes. ${ }^{4}$

In summary, one should consider the diagnosis of APT in the presence of three or more of the following: new or worsening symptoms or signs, new abnormalities on chest imaging, a decline in the total lung capacity $>15 \%$ or $\mathrm{D}_{\mathrm{L}} \mathrm{CO}>20 \%$, presence of phospholipidosis in lung cells, a marked CD8+lymphocytosis in lavage fluid, a lung biopsy that reveals diffuse alveolar damage, organizing pneumonia, interstitial pneumonitis, or fibrosis, and improvement in respiratory status following withdrawal of the drug with or without steroid therapy. ${ }^{2}$

Treatment of APT consists primarily of discontinuing amiodarone. This can, however, lead to an increased risk of recurrence of life-threatening arrhythmias. One option is to substitute another antiarrhythmic medicine though it may have more negative inotropic and proarrhythmic effects. Another option is to withhold amiodarone for several days, and then, reduce the dosage to its lowest effective level, though pulmonary toxicity has been noted to still occur at such doses. Other options include radiofrequency ablation of a causative re-entry mechanism and implantation of an automatic cardioverter defibrillator. ${ }^{5}$ Despite the lack of controlled studies, there is 
growing evidence that corticosteroids are beneficial. The current recommendations are to start with an adequate dose of 0.75 to $1 \mathrm{mg} / \mathrm{kg}$ of prednisolone or equivalent. The initial dosage should then be maintained until definite clinical and radiographic response is obtained, otherwise consider increasing the dose. Tapering should be slow since recurrences have been described up to eight months of cessation of therapy. A reasonable estimate of duration of treatment is six months, more often one year, and patients should be monitored carefully after discontinuation of corticosteroids for possible recurrence. Clinical improvement and clearing of pulmonary opacities typically require one to three months. Toxic effects may continue and even temporarily progress because of its persistence in the lung and accumulation in fatty tissues leading to a long half-life of up to 45 days. ${ }^{2,4,5}$

\section{References}

1. Al-Khatib SM, LaPointe NM, Curtis LH, et al. Outpatient prescribing of antiarrythmic drugs from 1995 to 2000. Am J Cardiol. 2003;91(1):91-94.

2. Vassallo P, Trohman RG. Prescribing amiodarone: an evidence-based review of clinical indications. JAMA. 2007 Sep 19;298(11):1312-22

3. Martin, WJ, Rosenow, EC. Amiodarone pulmonary toxicity. Recognition and pathogenesis (Part 1). Chest 1988; 93:1067.

4. Camus P, Martin WJ 2nd, Rosenow EC 3rd. Amiodarone pulmonary toxicity. Clin Chest Med. 2004 Mar;25(1):65-75.

5. Jessurun GA, Boersma WG, Crijns HJ. Amiodarone-induced pulmonary toxicity. Predisposing factors, clinical symptoms and treatment. Drug Saf. 1998 May;18(5):339-44.

6. Martin WJ 2nd, Rosenow EC 3rd. Amiodarone pulmonary toxicity. Recognition and pathogenesis (Part 2). Chest. 1988 Jun;93(6):1242-8.

7. Vorperian VR, Havighurst TC, Miller S, January CT. Adverse Effects of Low Dose Amiodarone: A Meta-Analysis. J Am Coll Cardiol July1997; Vol 30, Iss 3:791-8.

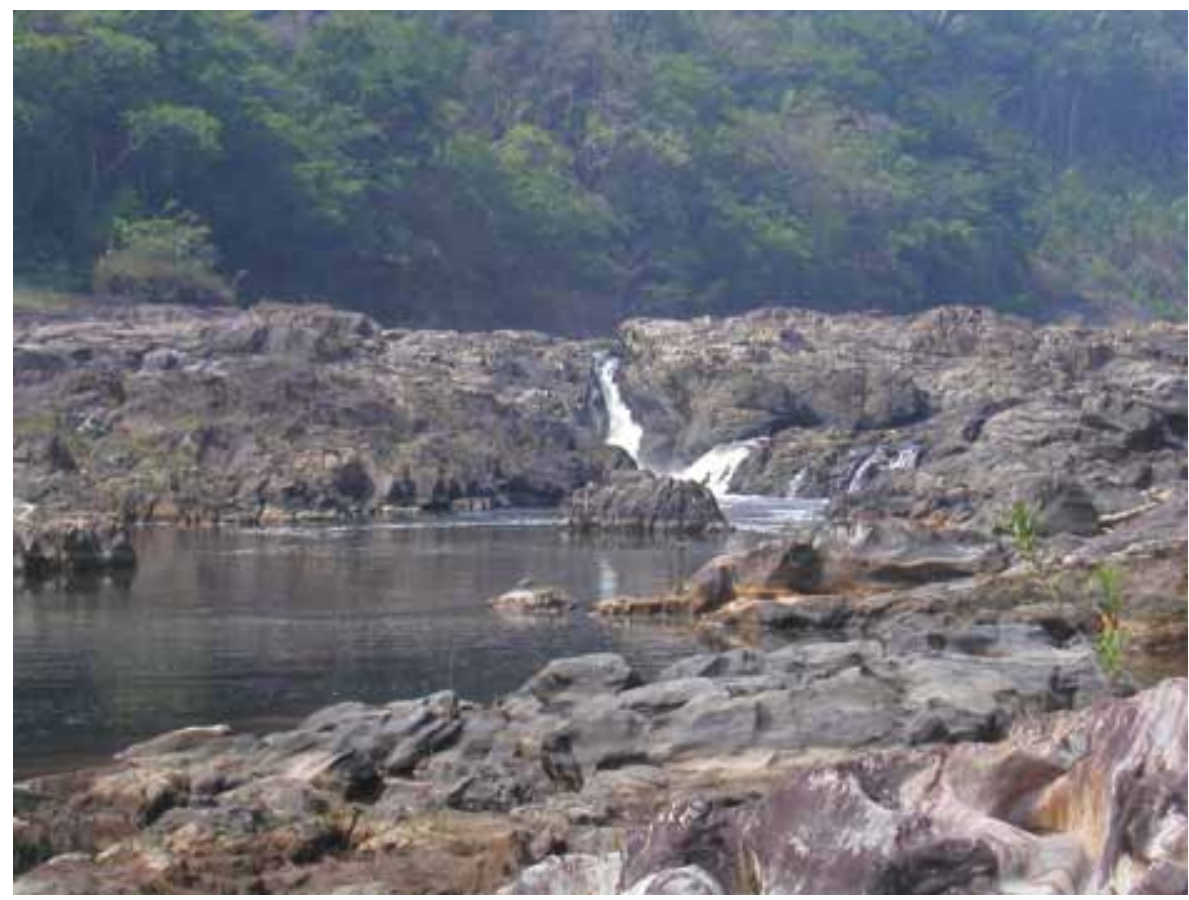

Photograph courtesy of Lisa Teng, MD 